\title{
Interactive comment on "Ages and transit times as important diagnostics of model performance for predicting carbon dynamics in terrestrial vegetation models" by Verónika Ceballos-Núñez et al.
}

Verónika Ceballos-Núñez et al.

vceball@bgc-jena.mpg.de

Received and published: 3 November 2017

We appreciate the time that Referee 4 dedicated to review our manuscript. In the text below we quote the referee's comments in italics and provide our response below in blue:

The article develops an interesting approach to distribute structural and labile carbon into age distributions with the resulting transit time of carbon in the vegetation. They Discussion paper tested three different allocation models and used measured carbon values from Har- 
vard Forest to parameterize each model and atmospheric isotopic carbon to compare against the isotopic signatures calculated from each structural component.

We are glad that the referee found the article interesting, but we have to clarify that we did not 'distributed the carbon into age distributions'; we calculated the age and transit time distribution of carbon particles in a vegetation system and its compartments.

Age and transit time distributions were different for the different allocation models, showing older age distribution in the model with more storage compartments, as well as with the wood components.

1. At the end of the introduction it is stated that the models are diagnosed according to four metrics, but only metrics 1 and 2 (carbon fluxes and bomb spike) are based on observations, and even those are not necessarily for each component, so the diagnosis is really just an analysis of how the different model results compare or what they imply rather than an actual metric based on observed values. There needs to be better distinction between what is actually observed $v s$ what is modeled throughout the paper.

We interpret this and the previous comment as a sign that we need to make our Methods section clearer. Please find enclosed the new version. The only measurements that we used for parameter optimization were published aboveground biomass, LMA and LAI. These measurements were only used in the optimization procedures, to find suitable parameter values. The model comparison was mainly qualitative, using simulations, e.g. the method used for the radiocarbon is clearly stated in the first line of the section 3.2.2. In the rest we discuss that we were comparing the model simulations with respect to their expected dynamics, depending whether they were fast or slow cycling components.

2. So, one justification for this research is that age and transit times can be measured with isotopic tracers, but that was not done in this study. There should be some examples in the literature of these types of measurements or some attempt to use them to

Printer-friendly version

Discussion paper
Interactive

comment

$\mathrm{C2}$ 
verify the results of this study. It would be nice if there were some way to prove that adding more compartments improves the age distribution and transit times. However, compared to the total atmospheric isotopic signatures, the multi-compartment schemes actually do worse, which the authors attribute to perhaps the lack of phenology.

In the Discussion we compared published mean ages of NSC with the ones predicted with the models. We agree that it would be more interesting to have measured $\mathrm{C}$ age distributions to compare with the simulations, but they are unfortunately unattainable with the current empirical methods. Also, one important clarification is that radiocarbon values for the different pools cannot be compared directly to atmospheric radiocarbon values as this comment seem to imply. They can only be compared to radiocarbon values measured on the tissue of the different pools.

3. The models used here are purely parameterized models without any processes at all, so how would this approach actually apply to models that were process-based? Bayesian optimization of parameters has been done before, but it is impressive that this approach was taken here. Any speculation about how this age and transit distribution approach could be applied and tested in some of the major ecosystem models?

The models we use are simple, which helps us to introduce the concepts of age and transit time of carbon in vegetation compartments. Independent on the complexity of the model being used, and the time steps of specific simulations, we can always expect mixes of $\mathrm{C}$ ages of different compartments, and this results in age and transit time distributions. The concept is independent on the complexity of the model, but the specific shape of the distributions would in fact depend on the specificity of the model. So, there is no consequence on whether the concept can or cannot be applied for more complex models, it only would affect the particular shape of the distributions.

4. Non structural carbon would seem to be the same thing as labile carbon, so maybe indicate that first time the term is used.

Thank you for this suggestion. We indicated that in the introduction. 
5. The allocation models here really refer to models with different quality storage pools. The allocation itself seems to be simply constant rates - but I would consider allocation model to imply different methods of determining allocation, i.e. literature-based rates, cost-benefit analysis, etc. I would prefer more clarity on how the constant rates differ between the pools, and how they are determined.

We agree that there are other methods to estimate the allocation rates, and that we did not explained the reasons why we chose to estimate the parameters of our models using the Bayesian optimization method. In the new version of the Methods section we mention that we chose this method because it gave us the possibility of exploring the parameter space and with that find parameter sets to do an uncertainty analysis of the model. The goal of the parameter estimation procedure was to obtain parameter sets for each model, which could allow them to simulate similar $C$ stocks to those obtained from the Harvard Forest Archives. The upper and lower boundaries used to constrain the parameter values during the estimation were obtained from the literature, for a similar forest. As we explained in the point 3, we decided to use simple models (with fixed coefficients) to illustrate the concept of 'age and transit time distributions'; this is why the environmental forcing was not part of the estimation of the rates. However, it is important to clarify that even if we would have used other methods to obtain the rates, we would have still obtained different ages and transit time distributions for each model'.

6. P. 7, and of first paragraph: How do the functions used to calculate age and transit time distributions relate to the formulas in the introduction? Last Paragraph: What is meant by lower-diagonal with respect to the figure?

The formulas mentioned in the introduction were implemented as functions in the ' $R$ ' package 'SoilR', in order to calculate age and transit time distributions. With "lowerdiagonal" we were referring to the values under the diagonal. We corrected that sentence.

Printer-friendly version

Discussion paper 
7. P. 9, Figure 3: Are the observations data the dots and vertical lines? Seems like a large range for the error bars - why so large, and what are they based on?

We agree that we did not explained properly the way in which we used the data obtained from the archives of Harvard Forest. We added a short explanation on how we used the measurements of aboveground biomass, LAI and LMA to calculate the C stocks that we show in figure 3 . The large deviations from the $C$ in wood is probably due to the large variation in the aboveground biomass measurements.

Table 2: What is the criteria for positive or negative correlations (i.e. how large and R2 value?)

The assignment of positive or negative correlations was performed based on the sign of the values observed in the pairwise plots. Only $R^{2}$ values $<-0.1$ and $>0.1$ were assumed to account for correlations.

8. Figure 4 is not mentioned in the text. More detail on the source of the isotopic carbon data would be useful. How are the "bomb spikes" used to determine radiocarbon values?

We had actually referenced the figure, but only with a number, which is probably the reason why it was overlooked. We corrected that mistake. The calculation of radiocarbon in the different compartments relies on a standard atmospheric radiocarbon curve (Hua et al. 2013), which is used to introduce radiocarbon in all vegetation compartments at rates specified by the vector $u$ and the transfer matrix $B$. This functionality is part of the 'SoilR' packaged used here. We added a short description in the methods about the incorporation of radiocarbon in the simulations and provided a citation for additional details of its implementation in the SoilR package.

9. P. 11: Not sure whatis meant by "Notice that distributions with the highest peak (occurred) closer to 0 years, and with younger mean and median ages had the youngest C".

Printer-friendly version

Discussion paper 
That sentence was meant to explain how to interpret the shape of the distributions and, based on that, show that the model Storage: 0 had younger $C$ than Storage: 1 and 2. However this explanation might not be needed, so we removed that sentence.

10. P. 16, second paragraph: What parameters are the ones that compose the beta vector and $B$ matrix?

The elements that compose the $\beta$ vector and the $\mathbf{B}$ matrix are described in the equation (1). The components of the matrix $\mathbf{B}$ are represented by the symbols next to the arrows in figure 2 , which are the parameters that control the $C$ transfer through the compartments.

Not all the appendix figures (A4 to A9) are mentioned in the text.

We appreciate this remark, we have added the missing references.

At bottom of page, what are implications of the different age values -i.e. how do the $1.71 / 2.14$ and $45 \mathrm{yr}$ relate to the 7.2 ?

This is the part that we mentioned in the response to the comment 2. We were comparing the simulated mean ages of the NSC to the ones obtained from literature. The published values had a wide range. Thus, although the mean ages of the storage compartments with fast cycling were lower than the mean published value, they were still within the range.

We hope that we addressed the comments of Referee 4 adequately and with that improved the clarity of this manuscript.

Please also note the supplement to this comment:

https://www.biogeosciences-discuss.net/bg-2017-308/bg-2017-308-AC4-

Printer-friendly version supplement.pdf

Discussion paper

Interactive comment on Biogeosciences Discuss., https://doi.org/10.5194/bg-2017-308, 2017. 